\title{
Psychosomatic Stress Responses and Sleep Disturbance Mediate the Effects of Irregular Mealtimes on Presenteeism
}

This article was published in the following Dove Press journal:

Neuropsychiatric Disease and Treatment

\author{
Taito Hayashida ${ }^{1,2}$ \\ Akiyoshi Shimura $\mathbb{D D}^{2}$ \\ Motoki Higashiyama ${ }^{2}$ \\ Yota Fujimura' \\ Kotaro Ono ${ }^{2}$ \\ Takeshi Inoue $\mathbb{I D}^{2}$ \\ 'Department of Psychiatry, Tokyo Medical \\ University Hachioji Medical Center, \\ Hachioji-shi, Tokyo, 193-0998, Japan; \\ ${ }^{2}$ Department of Psychiatry, Tokyo Medical \\ University, Shinjuku-ku, Tokyo, 160-0023, \\ Japan
}

Background: Presenteeism is the loss of work productivity of workers owing to physical and mental problems, and its socioeconomic effects are greater than those of absenteeism. Presenteeism is caused by psychological and physical dysfunctions. On the other hand, the regularity of mealtimes is an important factor associated with physical and mental health conditions. We hence assessed the association among the irregularity of mealtimes, presenteeism, psychological and physical stress responses, and sleep disturbance in office workers in companies.

Methods: From May to December 2017, the data of 2905 participants who had given their consent to use their information for academic purposes, and answered a questionnaire about the survey and their lifestyle were collected. Path analysis was performed to analyze the association between the irregularity of mealtimes, psychosomatic symptoms (Brief Job Stress Questionnaire), sleep disturbance (Pittsburgh Sleep Questionnaire), and presenteeism (Work Limitations Questionnaire).

Results: The direct effect of the irregularity of mealtimes on presenteeism was significant but weak. However, the irregularity of mealtimes had a strong effect on presenteeism indirectly through psychological and physical stress responses and sleep disturbance.

Conclusion: Psychological and physical stress responses and sleep disturbance were identified as mediating factors of the effects of irregularity of mealtimes on presenteeism.

Keywords: presenteeism, Work Limitations Questionnaire, WLQ, mealtimes, sleep disturbance, stress reaction, mediating effect

\section{Introduction}

Presenteeism is working in poor health conditions that should prompt rest and absence from work, and results in productivity loss. ${ }^{1,2}$ Presenteeism has been reported to affect the workforce and management of companies, to be associated with job productivity, and to play a potentially important role in economic development. $^{3-5}$ Research on presenteeism has shown that costs associated with the productivity loss caused by presenteeism are greater than the costs of treatments for the associated physical and mental health conditions. ${ }^{6}$ Mental illness, including major depression, has been reported to be associated with presenteeism. ${ }^{7}$ Presenteeism might result in a greater cost to a workplace than absenteeism, which is not coming to work owing to health issues, and is also an important issue in public health and occupational medicine. ${ }^{4}$
Department of Psychiatry, Tokyo Medical University, 6-7-I Nishishinjuku, Shinjukuku, Tokyo, 160-0023, Japan

Tel $+81-3-3342-61$ II

Fax +8I-3-3340-4499

Email sim@tokyo-med.ac.jp 
Factors, including job stress, lead to reduced productivity and affect presenteeism. ${ }^{8-11}$ In particular, psychosomatic symptoms and psychological and physical stress responses have a large effect on presenteeism, and it has also been reported that presenteeism is closely associated with sleep disturbances. ${ }^{12-14}$ Sleep quality and inadequate sleep duration are associated with presenteeism. ${ }^{12-19}$ In addition, the sleep-wake cycle and circadian rhythm dysfunction are considered to contribute to the incidence and severity of a wide range of clinical and pathological conditions, including sleep disorders, cancer, depression, metabolic syndrome, and inflammation..$^{20}$ Misalignments of sleep and mealtimes affect the functional connectivity of the amygdala in the resting state. ${ }^{21}$ Therefore, it is possible that the irregularity of mealtimes is associated with psychosomatic dysfunctions and subsequent presenteeism; however, to our knowledge, there are no reports in the literature to date that refer to the association between mealtime irregularities and presenteeism.

In this study, we hypothesized that irregularities of mealtimes could affect presenteeism through various factors, such as sleep disturbances and psychological and physical stress responses, regarding the factors that were pointed out as described above. Therefore, the purpose of this study was to verify our hypothesis using path analysis on the data obtained from office workers.

\section{Methods}

\section{Participants}

From May to December 2017, a questionnaire was distributed to 3314 office workers of 17 offices from companies that agreed to join the survey and to the use of the data for academic purposes. Finally, 2905 participants (87.7\%; 1837 males and 1062 females; average age: 36.6 \pm 9.4 years) were included in the study. Statistical analysis was conducted on the data of the questionnaire responses on occupational stress, lifestyle habits, and presenteeism. The surveyed establishments belonged to tertiary industries, such as the service industry, finance industry, Information Technology industry, broadcasting industry, retail industry, public service sector, etc., and were in Tokyo. They did not include primary and secondary industries, such as agriculture, forestry and fisheries, and mining. There were no particular inclusion/exclusion criteria. This study was conducted with the approval of the Tokyo Medical University Medical Ethics Review Board (study approval no.: SH3652), and all data were anonymized. All of the participants gave their informed consent and the study was conducted in accordance with the Declaration of Helsinki.

\section{Questionnaires}

Demographic Characteristics and Mealtime Patterns

Demographic characteristics, such as age, sex, changes in work content, changes in life, overtime hours per month, and one-way commuting time were analyzed. Regarding mealtimes, the following 4-point Likert scale was used: "1: quite regular", "2: tend to be regular", "3: tend to be irregular", and "4: quite irregular", of which 1 and 2 were defined as no irregularity of mealtimes, and 3 and 4 as irregularity of mealtimes.

\section{Psychological and Physical Stress Responses}

The Brief Job Stress Questionnaire (BJSQ), which measures occupational work stressors, psychological and physical stress responses, social support, and job and life satisfaction was used for the evaluation of psychosomatic symptoms. ${ }^{22,23}$ The questionnaire asks about the condition of the participant in the previous month, and psychological and physical stress responses were evaluated using 29 items on a Likert scale of 1 to 4 , and calculated as the following 6 subscores: lack of vigor, irritability, fatigue, anxiety, depression, and somatic symptoms.

\section{Pittsburgh Sleep Quality Index (PSQI)}

PSQI is a questionnaire that analyzes sleep disturbance, and the validated Japanese Pittsburgh Sleep Quality Index was used in this study. ${ }^{24,25}$ The following 7 component scores are generated from 19 individual items: subjective sleep quality, sleep latency, sleep duration, habitual sleep efficiency, sleep disturbances, use of sleeping medication, and daytime dysfunction. In the Japanese version, 6 or more points of the summed score (global score) indicates the existence of sleep disturbance. ${ }^{24,25}$

\section{Work Limitations Questionnaire (WLQ)}

WLQ is a questionnaire that asks about the influence of health issues on work, and evaluates presenteeism. The short form and the validated Japanese version was used. ${ }^{26,27}$ WLQ consists of the following components: physical demands, which covers the ability to perform tasks involving body strength, movement, endurance, coordination, and flexibility; time management, which addresses difficulty handling the time and scheduling demands of a job; mental-interpersonal demands, which addresses cognitively demanding tasks and on-the-job social interactions; 
and output demands, which addresses reduced work quantity, quality, and timeliness. From the WLQ, the percentage of productivity loss owing to presenteeism can be calculated.

\section{Data Analysis Model}

The hypothesis was that the irregularity of mealtimes enhances psychological and physical stress responses, sleep disturbances, and presenteeism. It was also assumed that the irregularity of mealtimes indirectly affects presenteeism through psychological and physical stress responses and sleep disturbances. Based on such a hypothesis, a structural equation model was created, as shown in Figure 1. Controls were not included in this analysis.

\section{Statistical Analysis}

Statistical analysis was performed using IBM SPSS Statistics ver. 26 and AMOS ver. 22 software. The structural equation model was analyzed by robust maximum likelihood estimation in covariance structure analysis. As the model in this study was a saturation model, a goodness-of-fit index was not used. The coefficients of the covariance structure analysis were standardized. The Pearson correlation coefficient and the $t$-test were performed using SPSS Statistics ver. 26 software to compare demographic information and questionnaire data. The objective variable was WLQ, and the explanatory variables were demographic information and each score (age, sex, overtime work hours per month, oneway commuting time, irregularity of mealtimes, psychological and physical stress response of BJSQ, global PSQI score,

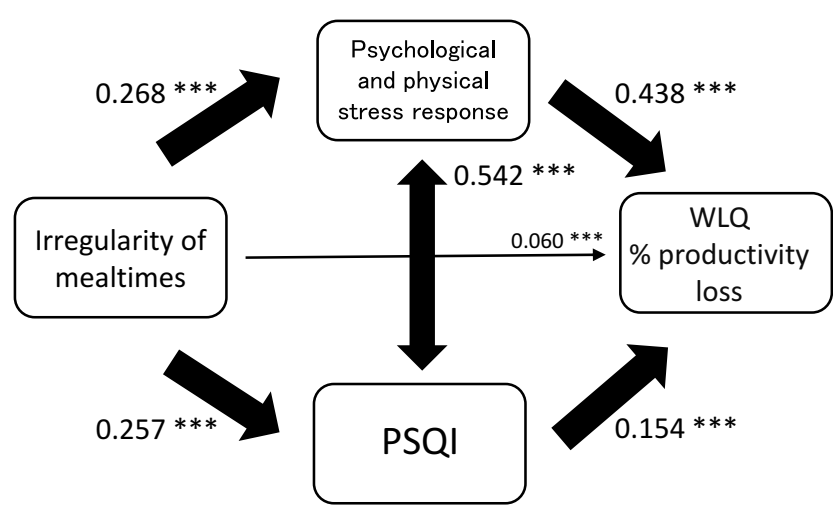

Figure I Results of covariance structure analysis of the structural equation model with the scores of the irregularity of mealtimes (no $=0$, yes $=1$ ), psychological and physical stress responses, the Japanese Pittsburgh Sleep Quality Index (PSQI), and Work Limitations Questionnaire (WLQ) percent productivity loss of 2905 nonclinical self-selected adult volunteers. Rectangles indicate the observed variables. Arrows with solid lines represent statistically significant paths. Numbers beside the arrows show direct standardized path coefficients. Indirect effects through the variables are described in the Results section. ${ }^{* * *} p<0.001$.
WLQ percent productivity loss). Multiple regression analysis was performed by the forced entry method.

\section{Results}

\section{Correlation Between Basic Information, Questionnaire Data, and Presenteeism of All Survey Participants}

Table 1 shows the association between demographic data, questionnaire data, and presenteeism of the 2905 general office workers at the time of the survey. Presenteeism was used as an objective variable, and irregularity of mealtimes, psychological and physical stress responses, and sleep disturbances were used as the explanatory variables. In the univariate analysis for each item, "age" and "female" were significantly associated with low WLQ percent productivity loss (Table 1). On the other hand, "changes in work content within the previous year", "changes in life within the previous year", "overtime work hours per month", "PSQI global score", "psychological and physical stress response", and "irregularity of mealtimes" were significantly associated with high WLQ percent productivity loss (Table 1).

\section{Multiple Regression Analysis on Presenteeism}

Table 2 shows the results of multiple regression analysis on presenteeism $(F=159.4, p<0.001)$. The 9 explanatory variables were added to the analysis, and the factors that had a significant effect on WLQ percent productivity loss were "age", "sex", "changes in work content within the previous year", "PSQI global score", "psychological and physical stress response", and "irregularity of mealtimes". The other variables did not have a statistically significant effect.

\section{Path Analysis}

Figure 1 shows the results of the structural equation model created by robust maximum likelihood estimation using irregularity of mealtimes (no $=0$, yes $=1$ ), psychological and physical stress responses, sleep disturbances, and presenteeism as observed variables. Regarding a direct effect, irregularity of mealtimes on presenteeism showed a significant but weak effect (standardization path coefficient: $0.060, p=0.001)$. On the other hand, the path from psychological and physical stress responses to presenteeism $(0.438, p<0.001)$ and from sleep disturbances to 
Table I Characteristics, Regularity of Meal Times, and Psychological and Physical Stress Responses Evaluated Using BJSQ, PSQI Global Score, and the Correlation with WLQ Percent Productivity Loss Score

\begin{tabular}{|c|c|c|}
\hline $\begin{array}{l}\text { Characteristics } \\
\text { or Measures }\end{array}$ & $\begin{array}{l}\text { Number or } \\
\text { Mean } \pm \text { SD }\end{array}$ & $\begin{array}{l}\text { Correlation with WLQPLS (r) or Effect on WLQPLS } \\
\text { (mean } \pm \text { SD of WLQPLS, } t \text {-Test) }\end{array}$ \\
\hline Age & $36.6 \pm 9.39$ & $r=-0.11, p<0.001$ \\
\hline Sex (male: female) & I,837: 1,062 & $\begin{array}{l}\text { Male } 5.9 \% \pm 4.6 \% \text { vs } \\
\text { female } 5.4 \% \pm 4.4 \% \text { (t-test), } \\
p=0.010\end{array}$ \\
\hline $\begin{array}{l}\text { Changes in work content } \\
\text { within the previous year (yes: no) }\end{array}$ & I,592: 1,313 & $\begin{array}{l}\text { Yes } 6.2 \% \pm 4.7 \% \text { vs } \\
\text { no } 5.1 \% \pm 4.3 \% \text { ( } t \text {-test), } p<0.001\end{array}$ \\
\hline $\begin{array}{l}\text { Changes in life within the previous } \\
\text { year (yes: no) }\end{array}$ & I,069: 1,836 & $\begin{array}{l}\text { Yes } 6.1 \% \pm 4.7 \% \text { vs } \\
\text { no } 5.5 \% \pm 4.4 \% \text { ( } t \text {-test), } p=0.002\end{array}$ \\
\hline Overtime work per month (hours) & $23.6 \pm 26.5$ & $r=0.040, p=0.031$ \\
\hline One-way commuting time (minutes) & $54.5 \pm 24.7$ & $r=0.023, p=0.21$ \\
\hline PSQI global score (points) & $6.4 \pm 2.8$ & $r=0.42, p<0.001$ \\
\hline Psychological and physical stress responses of BJSQ (points) & $56.7 \pm 13.9$ & $r=0.54, p<0.001$ \\
\hline Irregularity of mealtimes (yes: no) & I,I59: I,746 & $\begin{array}{l}\text { Yes } 6.6 \% \pm 4.3 \% \text { vs } \\
\text { no } 4.7 \% \pm 3.9 \% \text { ( } t \text {-test), } p<0.00 \text { I }\end{array}$ \\
\hline WLQ \%productivity loss score & $5.5 \% \pm 4.2 \%$ & \\
\hline
\end{tabular}

Notes: Data are presented as means \pm SD or numbers; $r$, Pearson correlation coefficient.

Abbreviations: BJSQ, Brief Job Stress Questionnaire; PSQI, Pittsburgh Sleep Quality Index; WLQ, Work Limitations Questionnaire; WLQPLS, WLQ productivity loss score.

Table 2 Results of Multiple Regression Analysis of WLQ Percent Productivity Loss Score

\begin{tabular}{|l|l|l|l|}
\hline Selected Independent Factors & Standardized Partial Regression Coefficient (Beta) & $p$-value & $<0.001$ \\
\hline $\begin{array}{l}\text { Psychological and physical } \\
\text { stress response of BJSQ }\end{array}$ & 0.450 & $<$ VIF \\
\hline PSQI global score & 0.145 & $<.571$ \\
\hline Sex & -0.115 & $<0.001$ & 1.525 \\
\hline Age & -0.075 & $<0.001$ & 1.089 \\
\hline Irregularity of meal times & 0.048 & 0.004 & 1.096 \\
\hline $\begin{array}{l}\text { Changes in work content } \\
\text { within the previous year }\end{array}$ & 0.036 & 0.022 \\
\hline Overtime work hours per month & -0.028 & 1.150 \\
\hline Changes in life & -0.004 & 1.078 \\
\hline One-way commuting time & 0.003 & 0.082 \\
\hline Adjusted $R^{2}=0.329$ & $F=158.534$ & 0.805 & 1.083 \\
\hline
\end{tabular}

Notes: Dependent factor: WLQ percent productivity loss score. Independent factors: age, sex (male $=0$, female $=1$ ), changes in work content $(n o=0$, yes $=1$ ), changes in life $($ no $=0$, yes $=1$ ), overtime work hours per month, one-way commuting time, PSQI global score, psychological and physical stress responses of BJSQ, irregularity of mealtimes $($ no $=0$, yes $=1$ ).

Abbreviations: Beta, standardized partial regression coefficient; VIF, variance inflation factor; PSQI, Pittsburgh Sleep Quality Index global score; WLQ, Work Limitations Questionnaire. 
presenteeism $(0.154, p<0.001)$ were highly significant. Regarding indirect effects, the irregularity of mealtimes was found to significantly affect presenteeism indirectly through psychological and physical stress responses and sleep disturbances $(0.117, p<0.001$ via psychological and physical stress responses and $0.039, p<0.001$ via sleep disturbances, respectively), and the total indirect effect was greater than the direct effect. The total standardized effect from the irregularity of mealtimes to presenteeism was 0.216 . This model also accounted for $31.5 \%$ of the variability in general office worker presenteeism, because the square of the multiple correlation coefficient $\left(R^{2}\right)$ of the summary score of the WLQ percent productivity loss in this model was 0.315 . Because the sum of the indirect effects was much larger than that of the direct effects, the results indicated partial mediation, which was very close to full mediation.

\section{Discussion}

In this study, the irregularity of mealtimes was found to indirectly affect presenteeism through psychological and physical stress responses and sleep disturbances. The direct effect of the irregularity of mealtimes on presenteeism was significant but weak. A previous study reported that work stressors and problems with surrounding support reduced productivity through sleep disturbances and psychological and physical stress responses. ${ }^{12}$ Our present study suggests a mediating effect of the irregularity of mealtimes on presenteeism through psychological and physical stress responses and sleep disturbance. Our results also indicated that the irregularity of mealtimes affects sleep, as has been reported in previous studies. ${ }^{13,28}$ In addition, our study suggested that the irregularity of mealtimes also affects the psychological and physical stress responses of occupational stress. However, we found no reports in the literature to date regarding the effects of the irregularity of mealtimes on the psychosomatic stress response of occupational stress.

The mechanism by which the irregularity of mealtimes results in psychosomatic symptoms and sleep disturbances is presently unclear, although there are some hypotheses. Regarding metabolic aspects, the irregularity of mealtimes leads to a deterioration in blood glucose and insulin profiles. ${ }^{29,30}$ Regarding circadian rhythm, regular mealtimes reset an animal's internal clock system, and might be associated with stress responses and sleep. ${ }^{31-33}$ Irregular mealtimes might have a negative effect on blood glucose and insulin, and result in a failure to reset the biological clock, which would then cause sleep disturbances and affect presenteeism.

This study suggested that the irregularity of mealtimes might cause presenteeism indirectly through pathways that influence sleep disturbances and psychological and physical stress responses. Improving presenteeism is important from public, occupational, and socioeconomic perspectives. The results of this study suggest that specific measures to encourage regular mealtimes would improve worker productivity. To our knowledge, no study to date has shown an association between the irregularity of mealtimes and psychological and physical stress responses, sleep disturbances, and presenteeism, and our present study has shown these associations for the first time.

There were five limitations to this study. First, the study was based on a questionnaire and relied on the memories of the participants, so there might be some inaccuracies. Second, the participants in this study were office workers, not the general population. Thirdly, this study had a cross-sectional design and was not a prospective study, and hence a long-term, prospective follow-up study should be performed in the future. Fourthly, the measures used to assess mealtime irregularities were created by ourselves, and were not validated. Future studies should hence be performed using validated measures. Finally, presenteeism in this study was evaluated using the WLQ, which measures both behaviors and outcomes at work. According to a previous meta-analysis, this may result in a bias of the overestimation of presenteeism. ${ }^{34}$

\section{Conclusion}

The results of this study suggest that the irregularity of mealtimes cause presenteeism indirectly through pathways of sleep disturbances and their effects on psychological and physical stress responses. Therefore, regular mealtimes are expected to improve worker presenteeism.

\section{Author Contributions}

All authors made substantial contributions towards the conception and design of the study, acquisition of data, or analysis and interpretation of the data; took part in drafting the manuscript or revising it critically for important intellectual content; agreed to its submission to the present journal; gave final approval of the version to be published; and agree to be accountable for all aspects of the work. 


\section{Funding}

This work was partly supported by a Grant-in-Aid for Scientific Research (no. 20K07955 to A. Shimura) from the Japanese Ministry of Education, Culture, Sports, Science and Technology.

\section{Disclosure}

Akiyoshi Shimura has received fees from Meiji Seika Pharma, Yoshitomi Yakuhin, Tanabe Mitsubishi Pharma, and Eisai outside of the submitted work. Yota Fujimura has received research/grant support from Novartis Pharma, Otsuka Pharmaceutical, Astellas, Dainippon Sumitomo Pharma, and Shionogi. Takeshi Inoue has received personal fees from Mochida Pharmaceutical, Takeda Pharmaceutical, Eli Lilly, Janssen Pharmaceutical, Merck Sharp and Dohme, Taisho Toyama Pharmaceutical, Yoshitomiyakuhin, and Daiichi Sankyo; grants from Shionogi, Astellas, Tsumura, and Eisai; grants and personal fees from Otsuka Pharmaceutical, Dainippon Sumitomo Pharma, Mitsubishi Tanabe Pharma, Kyowa Pharmaceutical Industry, Pfizer, Novartis Pharma, and Meiji Seika Pharma; and is a member of the advisory boards of Pfizer, Novartis Pharma, and Mitsubishi Tanabe Pharma. The other authors declare that they have no actual or potential conflicts of interest associated with this study.

\section{References}

1. Aronsson G, Gustafsson K, Dallner M. Sick but yet at work. An empirical study of sickness presenteeism. J Epidemiol Community Health. 2000;54:502-509. doi:10.1136/jech.54.7.502.

2. Fujino Y. Health management of workers with presenteeism is a new challenge in occupational health. $J$ UOEH. 2018;40(3):225-230. doi:10.7888/juoeh.40.225

3. Brborović H, Daka Q, Dakaj K, et al. Antecedents and associations of sickness presenteeism and sickness absenteeism in nurses: a systematic review. Int J Nurs Pract. 2017;23:6. doi:10.1111/ijn.12598

4. Burton WN, Pransky G, Conti DJ, et al. The association of medical conditions and presenteeism. J Occup Environ Med. 2004;46(6 Suppl): S38-45. doi:10.1097/01.jom.0000126687.49652.44

5. Kigozi J, Jowett S, Lewis M, et al. The estimation and inclusion of presenteeism costs in applied economic evaluation: a systematic review. Value Health. 2017;20(3):496-506. doi:10.1016/j. jval.2016.12.006

6. Loeppke R, Taitel M, Haufle V, et al. Health and productivity as a business strategy: a multiemployer study. J Occup Environ Med. 2009;51(4):411-428. doi:10.1097/JOM.0b013e3181a39180

7. Goetzel RZ, Long SR, Ozminkowski RJ, et al. Health, absence, disability, and presenteeism cost estimates of certain physical and mental health conditions affecting U.S. employers. J Occup Environ Med. 2004;46 (4):398-412. doi:10.1097/01.jom.0000121151.40413.bd

8. Jeon SH, Leem JH, Park SG, et al. Association among working hours, occupational stress, and presenteeism among wage workers: results from the Second Korean working conditions survey. Ann Occup Environ Med. 2014;26(1):6. doi:10.1186/2052-4374-26-6
9. Umann J, Guido Lde A, Silva RM. Stress, coping and presenteeism in nurses assisting critical and potentially critical patients. Rev Esc Enferm USP. 2014;48(5):891-898. doi:10.1590/s00806234201400005000016.

10. Yang T, Guo Y, Ma M, et al. Job stress and presenteeism among Chinese healthcare workers: the mediating effects of affective commitment. Int $J$ Environ Res Public Health. 2017;14(9):978. doi:10.3390/ijerph14090978

11. Yang T, Ma M, Zhu M, et al. Challenge or hindrance: does job stress affect presenteeism among Chinese healthcare workers? J Occup Health. 2018;60(2):163-171. doi:10.1539/joh.17-0195OA.

12. Furuichi W, Shimura A, Miyama H, et al. Effects of job stressors, stress response, and sleep disturbance on presenteeism in office workers. Neuropsychiatr Dis Treat. 2020;27(16):1827-1833. doi:10.2147/NDT.S258508

13. Shimura A, Sugiura K, Inoue $M$, et al. Which sleep hygiene factors are important? comprehensive assessment of lifestyle habits and job environment on sleep among office workers. Sleep Health. 2020;6 (3):288-298. doi:10.1016/j.sleh.2020.02.001

14. Taya G, Shimura A, Ishibashi Y, et al. Factors measured in stress checks are associated with presenteeism. Psychiatry. 2020;62 (7): 1037-1043.

15. Ishibashi Y, Shimura A. Association between work productivity and sleep health: a cross-sectional study in Japan. Sleep Health. 2020;6 (3):270-276. doi:10.1016/j.sleh.2020.02.016.

16. Guertler D, Vandelanotte C, Short C, et al. The association between physical activity, sitting time, sleep duration, and sleep quality as correlates of presenteeism. J Occup Environ Med. 2015;57 (3):321-328. doi:10.1097/JOM.0000000000000355.

17. Espie CA, Pawlecki B, Waterfield D, et al. Insomnia symptoms and their association with workplace productivity: cross-sectional and pre-post intervention analyses from a large multinational manufacturing company. Sleep Health. 2018;4(3):307-312. doi:10.1016/j. sleh.2018.03.003

18. Swanson LM, Arnedt JT, Rosekind MR, et al. Sleep disorders and work performance: findings from the 2008 National Sleep Foundation Sleep in America poll. J Sleep Res. 2011;20(3):487-494. doi:10.1111/ j.1365-2869.2010.00890.x

19. Rosekind MR, Gregory KB, Mallis MM, et al. The cost of poor sleep: workplace productivity loss and associated costs. J Occup Environ Med. 2010;52(1):91-98. doi:10.1097/JOM.0b013e3181c78c30

20. Bechtold DA, Gibbs JE, Loudon AS. Circadian dysfunction in disease. Trends Pharmacol Sci. 2010;31(5):191-198. doi:10.1016/j. tips.2010.01.002

21. Yoncheva YN, Castellanos FX, Pizinger T, et al. Sleep and meal-time misalignment alters functional connectivity: a pilot resting-state study. Int J Obes (Lond). 2016;40(11):1813-1816. doi:10.1038/ ijo.2016.132.

22. Shimomitsu T. The Final Development of the Brief Job Stress Questionnaire Mainly Used for Assessment of the Individuals. Kato $M$ Ministry of Labour Sponsored Grant for the Prevention of WorkRelated Illness: The 1999 Report. 2000.

23. Ando E, Kawakami N, Shimazu A, et al. Reliability and validity of the English version of the New Brief Job Stress Questionnaire. In: 31 st International Conference on Occupational Health. Seoul, Korea, 31 May - 5 June, 2015. (31, 160621).

24. Buysse DJ, Reynolds CF 3rd, Monk TH, et al. The Pittsburgh Sleep Quality Index: a new instrument for psychiatric practice and research. Psychiatry Res. 1989;28(2):193-213. doi:10.1016/0165-1781(89) 90047-4

25. Doi Y, Minowa M, Uchiyama M, et al. Psychometric assessment of subjective sleep quality using the Japanese version of the Pittsburgh Sleep Quality Index (PSQI-J) in psychiatric disordered and control subjects. Psychiatry Res. 2000;97(2-3):165-172. doi:10.1016/s01651781(00)00232-8. 
26. Lerner D, Amick BC 3rd, Rogers WH, Malspeis S, Bungay K, Cynn D. The work limitations questionnaire. Med Care. 2001;39 (1):72-85. doi:10.1097/00005650-200101000-00009.

27. Takegami M, Yamazaki S, Greenhill A, et al. Work performance assessed by a newly developed Japanese version of the Work Limitation Questionnaire in a general Japanese adult population. J Occup Health. 2014;56(2):124-133. doi:10.1539/joh.13-0087-oa

28. Shimura A, Hideo S, Takaesu Y, et al. Comprehensive assessment of the impact of life habits on sleep disturbance, chronotype, and daytime sleepiness among high-school students. Sleep Med. 2018;44:12-18. doi:10.1016/j.sleep.2017.10.011

29. Alhussain MH, Macdonald IA, Taylor MA. Irregular meal-pattern effects on energy expenditure, metabolism, and appetite regulation: a randomized controlled trial in healthy normal-weight women. $\mathrm{Am}$ $J$ Clin Nutr. 2016;104(1):21-32. doi:10.3945/ajcn.115.125401

30. Farshchi HR, Taylor MA, Macdonald IA. Beneficial metabolic effects of regular meal frequency on dietary thermogenesis, insulin sensitivity, and fasting lipid profiles in healthy obese women. Am J Clin Nutr. 2005;81(1):16-24. doi:10.1093/ajcn/81.1.16
31. Tahara Y, Shibata S. Chronobiology and nutrition. Neuroscience. 2013;253:78-88. doi:10.1016/j.neuroscience.2013.08.049

32. Kim H, Jeong G, Park YK, Kang SW. Sleep quality and nutritional intake in subjects with sleep issues according to perceived stress levels. J Lifestyle Med. 2018;8(1):42-49. doi:10.15280/ jlm.2018.8.1.42.

33. Quick V, Shoff S, Lohse B, et al. Relationships of eating competence, sleep behaviors and quality, and overweight status among college students. Eat Behav. 2015;19:15-19. doi:10.1016/j. eatbeh.2015.06.012.

34. McGregor A, Sharma R, Magee C, et al. Explaining variations in the findings of presenteeism research: a meta-analytic investigation into the moderating effects of construct operationalizations and chronic health. J Occup Health Psychol. 2018;23(4):584-601. doi:10.1037/ ocp0000099
Neuropsychiatric Disease and Treatment

\section{Publish your work in this journal}

Neuropsychiatric Disease and Treatment is an international, peerreviewed journal of clinical therapeutics and pharmacology focusing on concise rapid reporting of clinical or pre-clinical studies on a range of neuropsychiatric and neurological disorders. This journal is indexed on PubMed Central, the 'PsycINFO' database and CAS, and
Dovepress

is the official journal of The International Neuropsychiatric Association (INA). The manuscript management system is completely online and includes a very quick and fair peer-review system, which is all easy to use. Visit http://www.dovepress.com/testimonials.php to read real quotes from published authors. 